\title{
Solve the Initial Value Problems of Fractional Order Using The Homotopy Analytical Method and Improve Them Using Pade' Approximations
}

\author{
Heba Sh. Mahmmood Kais I. Ibraheem \\ Department of Mathematics, College of Education Pure Science University of Mosul \\ ,Iraq \\ Mosul.nice.57@gmail.com \\ DOI: $10.33899 /$ edusj.1999.163332 \\ Received \\ 27/ 03 / 2018 \\ kaisismail@yahoo.com \\ Accepted \\ 26 / $06 / 2018$

\begin{abstract}
In this research, the initial value problems of the caputo- fractional order are solved using the homotopy analysis method. The Pade' approximation is added to the fractional order to improve this method. The improvement is confirmed through two examples by comparing the value of mean square error for homotopy analysis method and the improved method.
\end{abstract}

Key words : Homotopy Analysis Method (HAM), Pade' Approximation (PA), Mean Square Error(MSE). 
حل مسائل القيم الابتائية ذات الرتب الكسرية باستخدام طريقة هوموتوبي التحليلية وتحسينها باستخدام تقرببات بادي

\author{
هبة شكر محمود قيس اسماعيل ابراهيم \\ قسم الرياضيات ، كلية التربية للعلوم الصرفة ، جامعة الدوصل ، العراق \\ kaisismail@yahoo.com Mosul.nice.57@gmail.com
}

DOI: $10.33899 /$ edusj.1999.163332

القبول

الاستلام

$2018 / 06 / 26$

2018 / 03 / 27

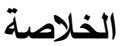

في هذا البحث تم حل مسائل القيم الابتدائية ذات الرتب الكسرية من نوع كابوتو باستعمال طريقة

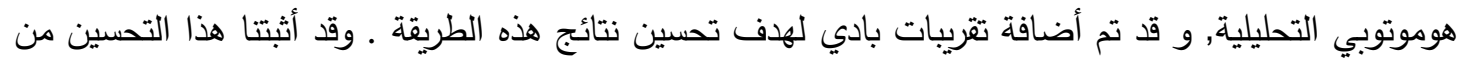

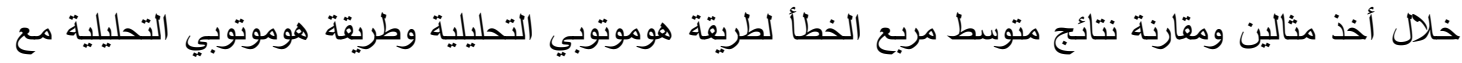
تقريبات بادي المان

الكلمات المفتاحية : طريقة هوموتوبي التحليلية , تقريبات بادي , متوسط مربع الخطا.

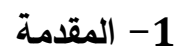

يعد موضوع حساب التفاضل والتكامل الكسري محط أهتمام الباحثين في أوائل عقد هذا القرن ومازال البحث عن هذا الموضوع مستمر [1,2,3,4,5 ]، بسبب استعمالاتها في مجالات عده منها الكهربائية, الانتشار ,

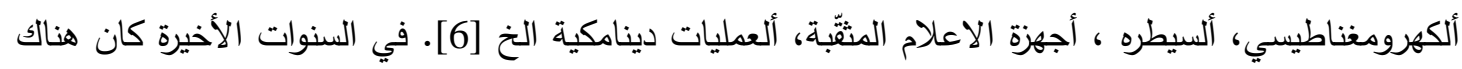

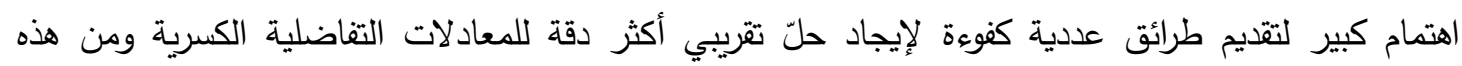
الطرائق، طريقة هوموتوبي التحليلية التي اقترحت اولا من قبل الباحث Liao في اطروحته للاكتوراه [7] التي لتئي

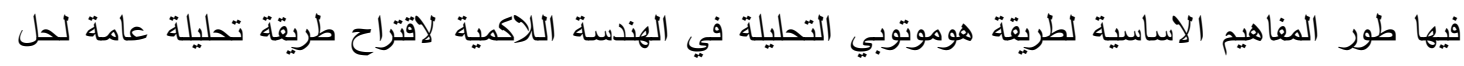
المشاكل غير الخطية. إن طريقة هوموتوبي التحليلية لاتعتمد على معلمة صغيرة على خلاف التقنيات التحليلية

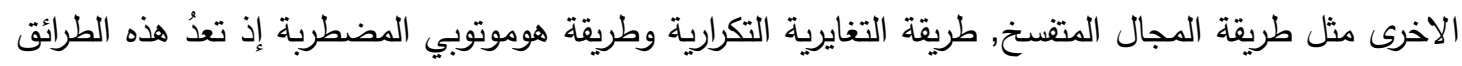

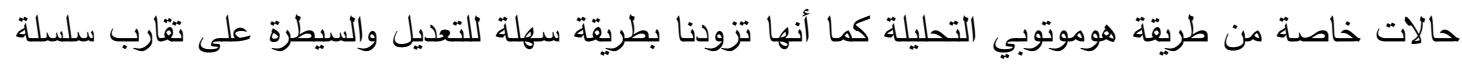
الحل من خلال التحكم بقيمة الخطأ عن طريق معلمة سيطرة التقارب h [8], هذة الطريقة حققت نجاحاً كبيراً

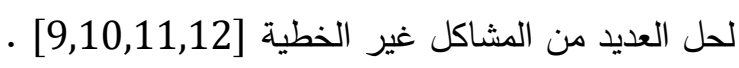


في هذا البحث استعملنا طريقة هوموتوبي التحليلية مع تقريبات بادي لحل مسائل القيم الابتدائية التي تحتوي

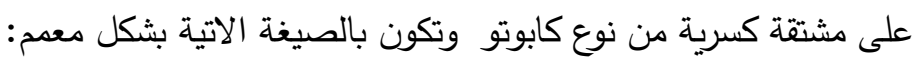

$N[y(t)]=D^{\alpha} y(t)+\delta y(t)-f(t)$

$y^{(i)}(t)=d_{i}$

$$
i=0,1, \cdots, n-1
$$

مع الثروط الابتدائية

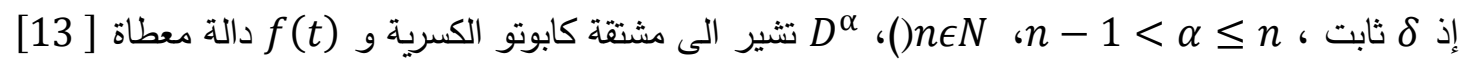

$$
2
$$

في هذا البند سنقدم بعض التعاريف الأساسية المرتبطة بالبحث

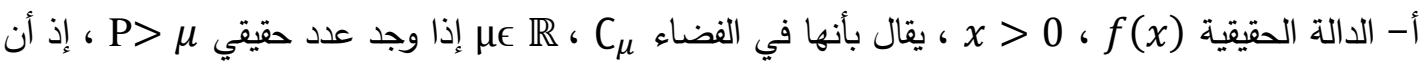

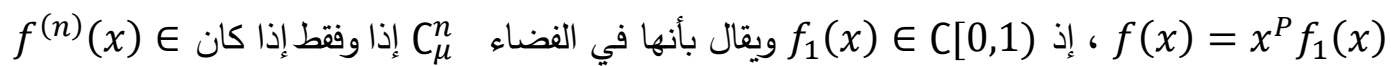
. [8] $n \in \mathbb{N}_{6} C_{\mu}$

ب- تكامل ريمان ليوفيل الكسري (Riemann-Liouvillel Fractional Integral) تكامل ريمان ليوفيل الكسري من الرتبة 0 > 0 للدالة $J^{\alpha} f(x)=\frac{1}{\Gamma(\alpha)} \int_{0}^{x}(x-t)^{\alpha-1} f(t) d t, x>0$,

$J^{0} f(x)=f(x)$

$$
\text { عندما } J^{\alpha}
$$

$$
\begin{aligned}
& J^{\alpha} J^{\beta} f(x)=J^{\beta} J^{\alpha} f(x) \\
& J^{\alpha} x^{\gamma}=\frac{\Gamma(\gamma+1)}{\Gamma(\alpha+\gamma+1)} x^{\alpha+\gamma}
\end{aligned}
$$

$\mu \geq-1, f \in C_{\mu}^{n}, n \in \mathbb{N}, n-1<\alpha \leq n$

$$
\begin{aligned}
& D_{*}^{\alpha} J^{\alpha} f(x)=f(x), x>0 . \\
& J^{\alpha} D_{*}^{\alpha} f(x)=f(x)-\sum_{K=0}^{n-1} f^{(k)}\left(0^{+}\right) \frac{x}{k !}, x>0 .
\end{aligned}
$$


ج-مشتقة كابوتو الكسرية(Caputo Fractional Devrative) [14]: لتكن f $\mathrm{f} \in \mathrm{C}_{\mu}$ : تعرف كالأتي : $D_{x}^{\alpha} f(x)=J^{n-\alpha} D^{n} f(x)=\frac{1}{\Gamma(n-\alpha)} \int_{0}^{x}(x-t)^{n-\alpha-1} f^{(n)}(t) d t$

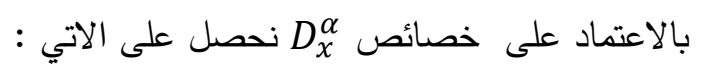

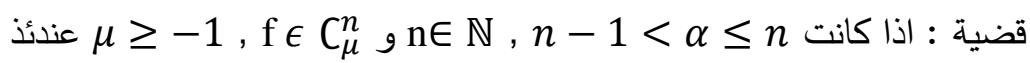

$D_{x}^{\alpha} D_{x}^{\beta} f(x)=D_{x}^{\alpha+\beta} f(x)=D_{x}^{\beta} D_{x}^{\alpha} f(x)$

$D_{x}^{\alpha} x^{\gamma}=\frac{\Gamma(1+\gamma)}{\Gamma(1+\gamma-\alpha)} x^{\gamma-\alpha}, x>0$

[15] (Pade' Approximate) د تقريبات بادي تقريب بادي للدالة متعددة الحدود (f) في الفترة [a,b] هو محاولة لتقليل قيمة الخطأ الاكبر في جزء من هذه الفترة إذ يعرف تقريب بادي للدالة (x) في الفترة [a,b) بأنه حاصل قسمة متعددات الحدود على $P_{N}(x)$

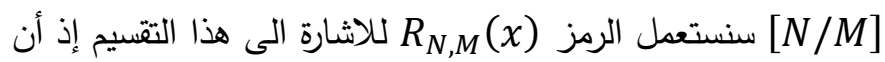

$R_{N, M}(x)=\frac{P_{N}(x)}{Q_{M}(x)}$

متعددات الحدود (x)

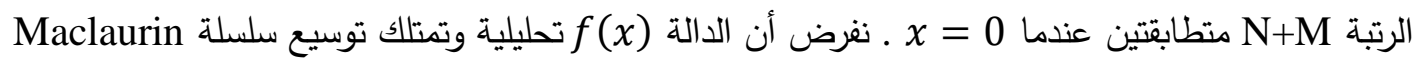
$f(x)=a_{0}+a_{1} x+a_{2} x^{2}+\cdots+a_{k} x^{k}+\cdots$

$$
\text { : } f(x) Q_{M}(x)-P_{N}(x)=Z_{N}(x) \text { (خبصيغة أخرى }
$$

$\left(\sum_{i=0}^{\infty} a_{i} x^{i}\right)\left(1+\sum_{i=1}^{M} q_{i} x^{i}\right)-\left(\sum_{i=0}^{N} p_{i} x^{i}\right)=\left(\sum_{i=0}^{\infty} c_{i} x^{i}\right)$

بتوسيع المعادلة (14) ومقارنة معاملات القوى بالنسبة لـ لـ الصى الصفر وذلك عندما تكون $0,1, \ldots, N+M$ ينتج معادلة خطية من الرتبة 1 ي لقيم . $Q_{M}(x)$ لها درجة أكبر بمقدار واحد من $P_{N}(x)$

[16] (MSE) Mean Square Error متوسط مربع الخطأ ليكن لدينا المتجهات المضبوط $\mathrm{MSE}=\frac{1}{N} \sum_{i=1}^{N}\left(E x\left(x_{i}\right)-\emptyset\left(x_{\mathrm{i}}\right)\right)^{2}$ 
3 [17 ط طريقة هوموتوبي التحليلية (HAM)

في هذا البند سوف نعطي بعض المفاهيم الاساسية لطريقة هوموتوبي التحليلية .

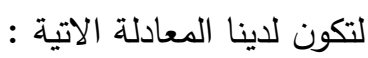

$N[y(t)]=0 \quad, \quad t \geq 0$

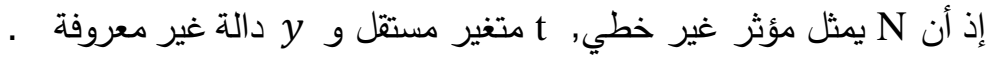
من خلال تعميم طريقة هوموتوبي التقليدية ثم إشتقاق معادلة التشوه من الرتبة الصفرية وصيغتها: $(1-q) \mathcal{L}\left[\varnothing(t, q)-y_{0}(t)\right]=q h H(t) N[\varnothing(t, q)]$

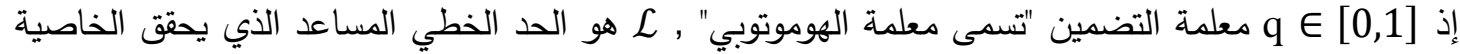

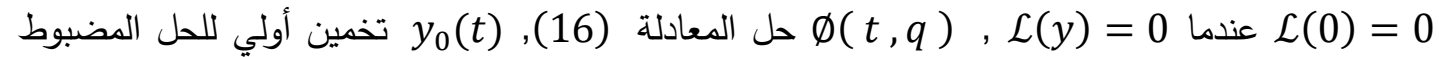
(أنه

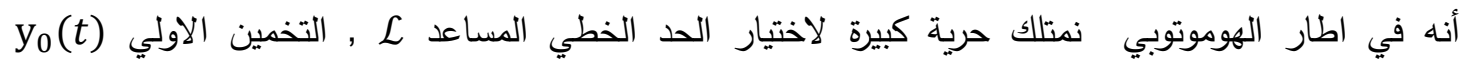
والمعلمات $\emptyset(t, 0)=y_{0}(t)$

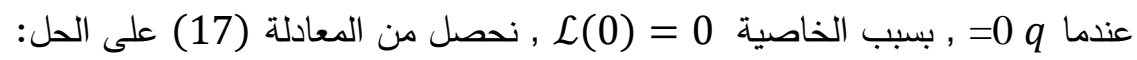
وعندما 1و= و بما أن 0 و $\emptyset(t, 1)=y(t)$

إذ (t) هو حل للمعادلة الاصلية (16) • وهكذا معلمة الهوموتوبي $q$ تتزايد من الصفر الى الواحد , الحل الت المستمر في الحل يسمى بالتشوه في الهوموتوبي. بتوسيع (t,q

$\emptyset(t, q)=y_{0}(t)+\sum_{m=1}^{+\infty} y_{m}(t) q^{m}$

$y_{m}(t)=\left.\frac{1}{m !} \frac{\partial^{m} \emptyset(t, q)}{\partial q^{m}}\right|_{\mathrm{q}=0}$

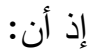
إذا كانت L 1

$y(\mathrm{t})=\emptyset(t, 1)=\mathrm{y}_{0}(t)+\sum_{m=1}^{+\infty} \mathrm{y}_{m}(t)$

$\vec{y}_{n}(t)=\left\{y_{0}(t), y_{1}(t), y_{2}(t), \ldots, y_{n}(t)\right\}$

باشتقاق معادلة التشويه(17) من الرتبة الصفرية m من المرات بالنسبة للمعلمة q ثم التعويض عن $q$ م بصفر واخيرا بتقسيم المعادلة على !m، نحصل على معادلة التثوه (deformation equation) التالية $\mathcal{L}\left[y_{m}(t)_{-} \chi_{m} y_{m-1}(t)\right]=h H(t) R_{m-1}\left(\vec{y}_{m-1}(t)\right)$ من الرتبة m: 
$R_{m-1}\left(\vec{y}_{m-1}(t)\right)=\left.\frac{1}{(m-1) !} \frac{\partial^{m-1} N[\varnothing(t, q)]}{\partial q^{m-1}}\right|_{\mathrm{q}=0}$

$\chi_{m}=\left\{\begin{array}{lc}0, & m \leq 1 \\ 1, & m>1\end{array}\right.$,

\section{4- تطبيق طريقة هوموتوبي التحليلية (HAM)}

في هذا البند سنستعمل طريقة هوموتوبي التحليلية مع تقريبات بادي لحل مسألة القيم الابتدائية (1)

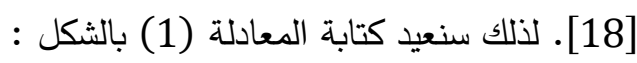
$N[y(t)]=0$

$N[y(t)]=D^{\alpha} y(t)+\delta y(t)-f(t)$

$$
y^{(i)}(t)=d_{i}, \quad i=0,1, \ldots, n-1
$$
سنعرف معادلة التشوه (deformation equation) من الرتبة الصفرية بالشكل:

$$
(1-q) D^{\alpha}\left[\varnothing(t, q)-y_{0}(t)\right]=q h H(t) N[\emptyset(t, q)] \alpha>0
$$

$$
\emptyset^{(i)}(0 ; q)=d_{i} \quad i=0,1, \ldots, n-1
$$

من الواضح عندما q=0 و (t) تحقق الثرط الابتدائي (29) و , نحصل على

$$
\emptyset(t, 0)=y_{0}(t)
$$

$$
\text { باستعمال معادلة التشوه (deformation equation) (24) من الرتبة m نحصل على : }
$$

$$
D^{\alpha}\left[y_{m}(t)_{-} \chi_{m} y_{m-1}(t)\right]=h H(t) R_{m-1}\left(\vec{y}_{m-1}(t)\right)
$$

$$
y_{m}{ }^{(i)}(0)=0 \quad i=0,1, \ldots, n-1
$$

إذ:

$$
R_{m-1}\left(\vec{y}_{m-1}(t)\right)=\left.\frac{1}{(m-1) !} \frac{\partial^{m-1}}{\partial q^{m-1}}\left(D^{\alpha} y(t)+\delta y(t)-f(t)\right)\right|_{q=0}
$$

بأخذ 1 بإ التكرارية الآتية : ب اخد

$$
D^{\alpha} y_{m}(t)=\chi_{m} D^{\alpha} y_{m-1}(t)+h R_{m-1}\left(\vec{y}_{m-1}(t)\right)
$$


الان نطبق تكامل ريمان ليوفيل J على طرفي المعادلة (36) نحصل على:

$y_{m}(t)=\chi_{m} y_{m-1}(t)-\chi_{m} \sum_{j=0}^{n-1} y_{m-1}{ }^{(j)}\left(0^{+}\right) \frac{t^{j}}{j !}$

$+h J^{\alpha}\left[R_{m-1}\left(\vec{y}_{m-1}(t)\right)\right] \quad m=1,2,3, \ldots$

بالتعويض عن قيم ...

بعد تعويضها مع (t) في المعادلة (22) نحصل على حل تقريبي لمسألة القيم الابتدائية (28) بالثرات $y(t)=\sum_{m=0}^{k} y_{m}(t)=\sum_{m=0}^{k} a_{m} t^{\beta_{m}}$

إذ أن الان نربط المتسلسلة (38) بتقرببات بادي وذلك باستعمال الفرضية الاتية:

$t^{\omega}=x$

إذ أن $\omega$ يمثل عدد كسري ،بعد التعويض في الحل التقريبي (38) نحصل على متسلسلة اسسها اعداد طبيعية

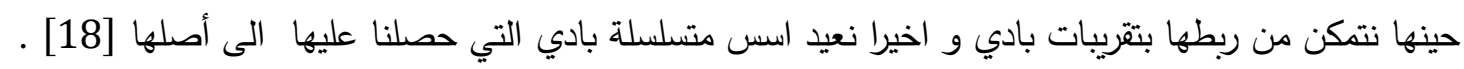
1.4 في هذا البند سنقوم بحل مثالين يوضحان كفاءة طريقة هوموتوبي التحليلية مع تقريبات بادي لحل مسائل القيم

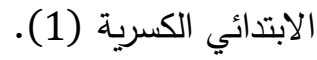
المثال(1) [19] لتكن لدينا مسألة القيم الابتدائية الخطية ذات الرتبة الكسرية $D^{\alpha} y(t)+y(t)=0, \quad 0<\alpha \leq 1, t \geq 0$ $y(0)=1$ و الحل المضبوط للمسألة (40)هو $y(t)=\sum_{K=0}^{\infty} \frac{\left(-t^{\alpha}\right)^{K}}{\Gamma(\alpha K+1)}$

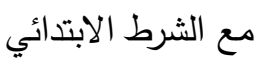
نفرض أن 1 = 1 و و بالاستعانة بالمعادلة (35) نستطيع تركيب هوموتوبي كآلاتي : $R_{m-1}\left(\vec{y}_{m-1}(t)\right)=D^{\alpha} y_{m-1}(t)+y_{m-1}(t)$

والآن باستعمال معادلة التشويه من الرتبة m m (37)عندما 1 تصبح: $y_{m}(t)=\chi_{m} y_{m-1}(t)-\chi_{m} \sum_{j=0}^{0} y_{m-1}^{(j)}\left(0^{+}\right) \frac{t^{j}}{j !} \alpha$

$+h J^{\alpha}\left[R_{m-1}\left(\vec{y}_{m-1}(t)\right)\right]$

$y_{1}(t)=h\left(\frac{t^{\alpha}}{\Gamma(\alpha+1)}\right)$ عندما 1 =1 1 , بالتعويض في المعادلة (41) نحصل على : 
وعندما ..

$y_{m}(t)=y_{m-1}(t)+h\left[y_{m-1}(t)+J^{\alpha} y_{m-1}(t)\right]$

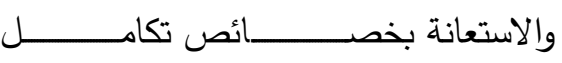

المعادل i $m=2$

$$
y_{2}(t)=y_{1}(t)+h\left[y_{1}(t)+J^{\alpha} y_{1}(t)\right]
$$

ريمان ليوفيل نحصل على :

$y_{2}(t)=h(1+h) \frac{t^{\alpha}}{\Gamma(\alpha+1)}+h^{2}\left(\frac{t^{2 \alpha}}{\Gamma(2 \alpha+1)}\right)$

بالتعويض عن $3=3$ في المعادلة (42) نحصل على:

$y_{3}(t)=y_{2}(t)+h\left[y_{2}(t)+J^{\alpha} y_{2}(t)\right]$

$y_{3}(t)=h(h+1)^{2} \frac{t^{\alpha}}{\Gamma(\alpha+1)}+2 h^{2}(h+1) \frac{t^{2 \alpha}}{\Gamma(2 \alpha+1)}+h^{3} \frac{t^{3 \alpha}}{\Gamma(3 \alpha+1)}$

وهكذا بتكرار هذه العملية نحصل على حل تقريبي (t) إذ أن:

$$
\begin{gathered}
y(t)=\sum_{m=0}^{5} y_{m}(t)=h\left[1+(1+h)+(1+h)^{2}+(1+h)^{3}+(1+h)^{4}\right] \frac{t^{\alpha}}{\Gamma(\alpha+1)} \\
+\cdots+h^{5} \frac{t^{5 \alpha}}{\Gamma(5 \alpha+1)}
\end{gathered}
$$

نربط متسلسلة الحل y(t) الناتجة بتقريبات بادي بعد التعويض عن قيم مختلفة لـ م ـ. الان بأخذ قيم مختلفة ل م وتعويضها في متسلسلة الحل (t) ثم ربطها بتقريبات بادي

بالتعويض عن $0.35=0.35$ =- 0.35 في متسلسلة الحل (43) نحصل على:

$y(t)=$

$-4.1468 \pi^{\frac{3}{2}} \sqrt{2} \mathrm{e}^{-4} t^{1.75}+7.9925 \pi \sqrt{3} \mathrm{e}^{-3} t^{1.40}-1.2980 \sqrt{\pi} \mathrm{e}^{-1} t^{1.05}+$

$6.2905 \mathrm{e}^{-1} t^{0.70}-9.9194 \mathrm{e}^{-1} t^{0.35}+1$

بأخذ قيم مختلفة دt وتعويضهم في متسلسلة الحل(44) نحصل على حلول تقريبية بطريقة هوموتوبي

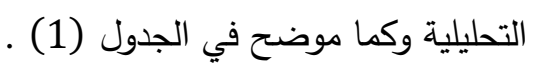
لحل المتسلسلة (44) بتقريبات بادي نفرض أن x = $x$ = $y^{*}(x)=-4.1468 \pi^{\frac{3}{2}} \sqrt{2} e^{-4} x^{5}+7.9925 \pi \sqrt{3} e^{-3} x^{4}-1.2980 \sqrt{\pi} e^{-1} x^{3}+$ 
$6.2905 e^{-1} x^{2}-9.9194 e^{-1} x^{1}+1$

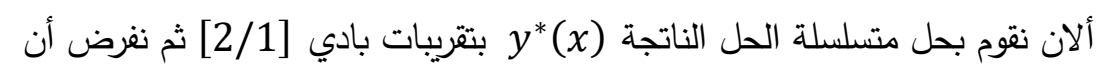

$$
[2 / 1]=\frac{1-6.2620 e^{-1} t^{0.35}+2.6627 e^{-1} t^{0.70}}{1+3.6573 e^{-1} t^{0.35}}
$$$$
\text { : } x=t^{0.35}
$$

بأخذ قيم مختلفة لـ t وتعويضهم في متسلسلة بادي (46) نحصل على حلول تقريبية بطريقة هوموتوبي التحليليلة

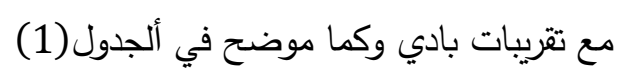

جدول (1): يتضمن نتائج الحل المضبوط , الحلول التقريبية بطريقة الهوموتوبي و الحلول التقريبية لكتسلسلة بادي (46) عند قيم t المختلفة.

\begin{tabular}{|c|c|c|c|}
\hline$t$ & Exact Solution & $\begin{array}{c}\text { Approximate } \\
\text { Solution using } \\
\text { Reliable HAM }\end{array}$ & $\begin{array}{c}\text { Approximate } \\
\text { Solution using } \\
\text { HAM-PA }\end{array}$ \\
\hline 0 & 1 & 1 & 1 \\
\hline 0.1 & $6.5490 \mathrm{e}^{-1}$ & $6.6359 e^{-1}$ & $6.6480 \mathrm{e}^{-1}$ \\
\hline 0.2 & $5.9584 e^{-1}$ & $6.0107 e^{-1}$ & $6.0401 \mathrm{e}^{-1}$ \\
\hline 0.3 & $5.5993 \mathrm{e}^{-1}$ & $5.6264 e^{-1}$ & $5.6755 \mathrm{e}^{-1}$ \\
\hline 0.4 & $5.3348 \mathrm{e}^{-1}$ & $5.3493 e^{-1}$ & $5.4196 \mathrm{e}^{-1}$ \\
\hline 0.5 & $5.1542 \mathrm{e}^{-1}$ & $5.1336 e^{-1}$ & $5.2262 \mathrm{e}^{-1}$ \\
\hline 0.6 & $5.010 \mathrm{e}^{-1}$ & $4.9577 e^{-1}$ & $5.0735 \mathrm{e}^{-1}$ \\
\hline 0.7 & $4.9067 \mathrm{e}^{-1}$ & $4.8098 e^{-1}$ & $4.9494 \mathrm{e}^{-1}$ \\
\hline 0.8 & $4.8398 \mathrm{e}^{-1}$ & $4.6826 e^{-1}$ & $4.8466 \mathrm{e}^{-1}$ \\
\hline 0.9 & $4.8102 \mathrm{e}^{-1}$ & $4.5714 e^{-1}$ & $4.7601 \mathrm{e}^{-1}$ \\
\hline 1.0 & $4.8198 \mathrm{e}^{-1}$ & $4.4727 e^{-1}$ & $4.6865 \mathrm{e}^{-1}$ \\
\hline
\end{tabular}

الجدول(2) يوضح الفرق بين متوسط مربع الخطأ بطريقة الهوموتوبي التحليلية للمتسلسلة (44) وطريقة هوموتوبي التحليلية مع تقريبات بادي [2/1].

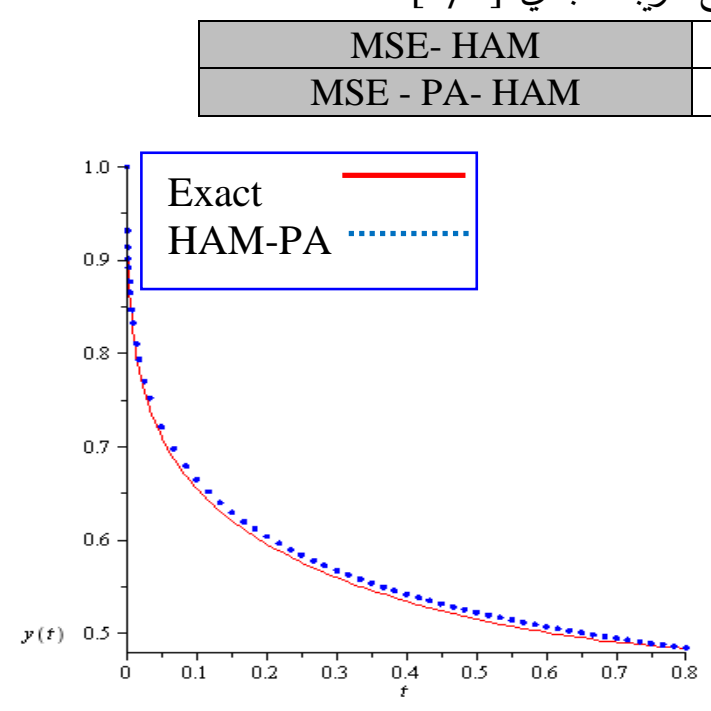

(b)

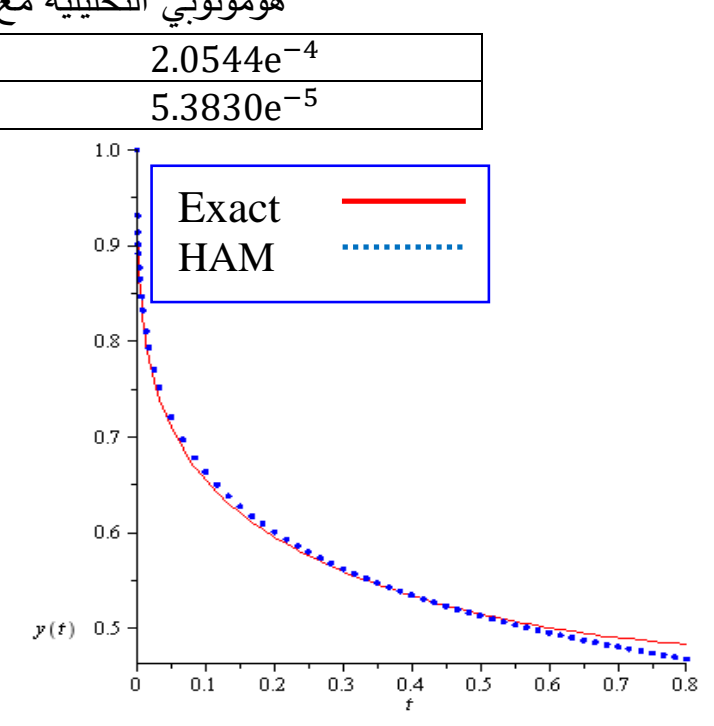

(a)

$$
\text { الثكل(1) : مقارنة بين الحل المضبوط والطرق المستخدمة عندما }
$$


(a) : الحل المضبوط مع طريقة هوموتوبي التحليلية

(b) : الحل المضبوط مع طريقة هوموتوبي التحليلية _ تقريبات بادي

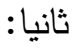

ثم بالتعويض عن $0.5=0.6$ = 0.6 في متسلسلة الحل (43) نحصل على:

$y(t)=-2.3398 \mathrm{e}^{-2} t^{2.5}+1.6848 \mathrm{e}^{-1} t^{2}-5.1345 \mathrm{e}^{-1} t^{1.5}+$

$9.2960 \mathrm{e}^{-1} t-1.1168 t^{0.5}+1$

بأخذ قيم مختلفة لـ وتعويضهم في متسلسلة الحل(47) نحصل على حلول تقريبية بطريقة هوموتوبي التحليلية

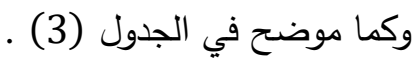

نفرض أن $x=x$ :

$y^{*}(x)=-2.3398 \mathrm{e}^{-2} x^{5}+1.6848 \mathrm{e}^{-1} x^{4}-$

$5.1345 \mathrm{e}^{-1} x^{3}+9.2960 \mathrm{e}^{-1} x^{2}-1.1168 x^{1}+1$

ألان نقوم بحل متسلسلة الحل الناتجة (48) بتقريبات بادي [4/3] ثم نفرض أن

$[4 / 3]=\frac{1-8.0636 e^{-1} t^{0.5}+6.4102 e^{-1} t-3.0315 e^{-1} t^{1.5}+6.575 e^{-2} t^{2}}{1+3.1042 e^{-1} t^{0.5}+7.477 e^{-2} t+1.030 e^{-2} t^{1.5}}$

بأخذ قيم مختلفة لـ t وتعويضهم في متسلسلة بادي (49) نحصل على حلول تقريبية بطريقة هوموتوبي التحليليلة

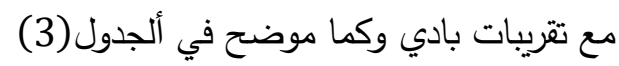

جدول (3): يتضمن نتائج الحل المضبوط , الحلول التقريبية بطريقة الهوموتوبي و الحلول التقريبية لمتسلسلة بادي (49)عند قيم t المختلفة.

\begin{tabular}{|c|c|c|c|}
\hline $\mathrm{T}$ & Exact Solution & $\begin{array}{c}\text { Approximate } \\
\text { Solution using HAM }\end{array}$ & $\begin{array}{c}\text { Approximate } \\
\text { Solution using } \\
\text { HAM-PA }\end{array}$ \\
\hline 0 & 1 & 1 & 1 \\
\hline 0.1 & $7.2357 e^{-1}$ & $7.2349 e^{-1}$ & $7.2350 e^{-1}$ \\
\hline 0.2 & $6.4378 e^{-1}$ & $6.4352 e^{-1}$ & $6.4354 e^{-1}$ \\
\hline 0.3 & $5.9202 e^{-1}$ & $5.9181 e^{-1}$ & $5.9183 e^{-1}$ \\
\hline 0.4 & $5.5362 e^{-1}$ & $5.5353 e^{-1}$ & $5.5354 e^{-1}$ \\
\hline 0.5 & $5.23211 e^{-1}$ & $5.2321 e^{-1}$ & $5.2322 e^{-1}$ \\
\hline 0.6 & $4.9818 e^{-1}$ & $4.9818 e^{-1}$ & $4.9828 e^{-1}$ \\
\hline 0.7 & $4.7706 e^{-1}$ & $4.7691 e^{-1}$ & $4.7698 e^{-1}$ \\
\hline 0.8 & $4.5899 e^{-1}$ & $4.5848 e^{-1}$ & $4.5858 e^{-1}$ \\
\hline 0.9 & $4.4347 e^{-1}$ & $4.4224 e^{-1}$ & $4.4238 e^{-1}$ \\
\hline 1.0 & $4.3005 e^{-1}$ & $4.2775 e^{-1}$ & $4.2796 e^{-1}$ \\
\hline
\end{tabular}


الجدول(4) يوضح الفرق بين متوسط مربع الخطأ بطريقة الهوموتوبي التحليلية للمتسلسلة (47) وطريقة

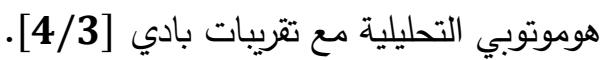

\begin{tabular}{|l|r|}
\hline MSE- HAM & $6.4457 e^{-7}$ \\
\hline MSE - PA- HAM & $5.4329 e^{-7}$ \\
\hline
\end{tabular}

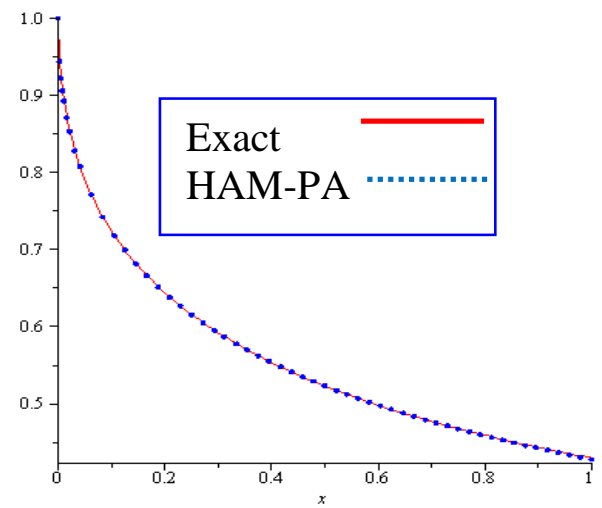

(b)

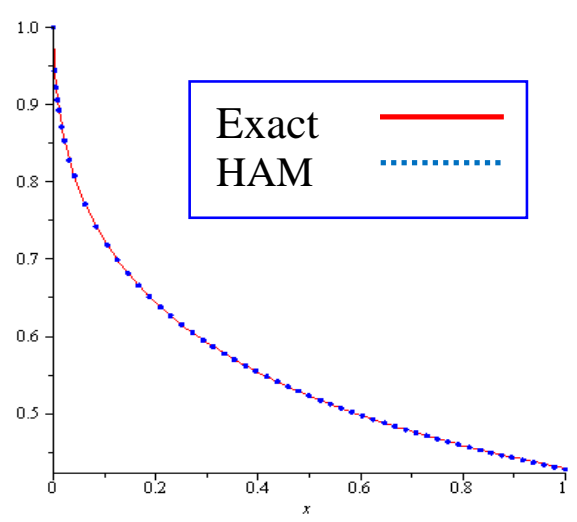

(a)

الثكل(2) : مقارنة بين الحل المضبوط والطرق المستخدمة عندما

(a) : الحل المضبوط مع طريقة هوموتوبي التحليلية

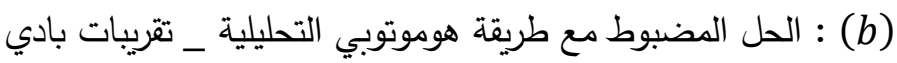

ثالثا:

بالتعويض عن $0.75=0.5$ = 0.5 في متسلسلة الحل (t) 1 نحصل على:

$y(t)=-2.3925 \mathrm{e}^{-4} \pi^{\frac{3}{2}} \sqrt{2} t^{3.75}+5.7430 \mathrm{e}^{-3} \pi \sqrt{3} t^{3}-1.1065 \mathrm{e}^{-1} \sqrt{\pi} t^{2.25}+$

$6.1120 \mathrm{e}^{-1} t^{1.5}-1.0540 t^{0.75}+1 \quad$ (50)

بأخذ قيم مختلفة دt وتعويضهم في متسلسلة الحل (t) نحصل على حلول تقريبية بطريقة هوموتوبي

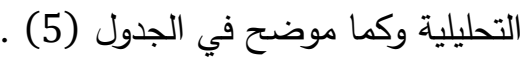

لحل المتسلسلة (50) نفرض أن $x$ =

$\mathrm{y}^{*}(x)=-2.3925 \mathrm{e}^{-4} \pi^{\frac{3}{2}} \sqrt{2} x^{5}+5.7430 \mathrm{e}^{-3} \pi \sqrt{3} x^{4}-1.1065 \mathrm{e}^{-1} \sqrt{\pi} x^{3}+$

$6.1120 \mathrm{e}^{-1} x^{2}-1.0540 x^{1}+1$ 
الان نقوم بحل متسلسلة الحل الناتجة (x) بتقريبات بادي [2/2 بم فرض أن

$$
[2 / 2]=\frac{1-5.3294 e^{-1} t^{0.75}+1.7801 e^{-1} t^{1.5}}{1+5.2111 e^{-1} t^{0.75}+1.1609 e^{-1} t^{1.5}}
$$

بأخذ قيم مختلفة لـ t وتعويضهم في متسلسلة بادي (52) نحصل على حلول تقريبية بطريقة

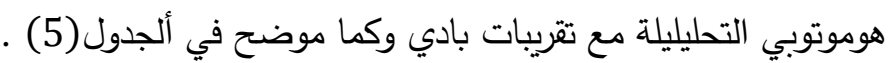

جدول (5): يتضمن نتائج الحل المضبوط , الحلول التقريبية بطريقة الهوموتوبي و الحلول التقريبية لمتسلسلة بادي (52)عند قيم $t$ المختلفة.

\begin{tabular}{|c|c|c|c|}
\hline$t$ & Exact Solution & $\begin{array}{c}\text { Approximate } \\
\text { Solution using } \\
\text { Reliable HAM }\end{array}$ & $\begin{array}{c}\text { Approximate } \\
\text { Solution using } \\
\text { HAM-PA }\end{array}$ \\
\hline 0 & 1 & 1 & 1 \\
\hline 0.1 & $8.2825 \mathrm{e}^{-1}$ & $8.3081 \mathrm{e}^{-1}$ & $8.3081 \mathrm{e}^{-1}$ \\
\hline 0.2 & $7.3258 \mathrm{e}^{-1}$ & $7.3442 \mathrm{e}^{-1}$ & $7.3444 \mathrm{e}^{-1}$ \\
\hline 0.3 & $6.6021 \mathrm{e}^{-1}$ & $6.6091 \mathrm{e}^{-1}$ & $6.6098 \mathrm{e}^{-1}$ \\
\hline 0.4 & $6.0212 \mathrm{e}^{-1}$ & $6.0144 \mathrm{e}^{-1}$ & $6.0161 \mathrm{e}^{-1}$ \\
\hline 0.5 & $5.5360 \mathrm{e}^{-1}$ & $5.5187 \mathrm{e}^{-1}$ & $5.5225 \mathrm{e}^{-1}$ \\
\hline 0.6 & $5.1228 \mathrm{e}^{-1}$ & $5.0980 \mathrm{e}^{-1}$ & $5.1050 \mathrm{e}^{-1}$ \\
\hline 0.7 & $4.7655 \mathrm{e}^{-1}$ & $4.7361 \mathrm{e}^{-1}$ & $4.7477 \mathrm{e}^{-1}$ \\
\hline 0.8 & $4.4529 \mathrm{e}^{-1}$ & $4.4218 \mathrm{e}^{-1}$ & $4.4396 \mathrm{e}^{-1}$ \\
\hline 0.9 & $4.1768 \mathrm{e}^{-1}$ & $4.1465 \mathrm{e}^{-1}$ & $4.1725 \mathrm{e}^{-1}$ \\
\hline 1.0 & $3.9312 \mathrm{e}^{-1}$ & $3.9037 \mathrm{e}^{-1}$ & $3.9400 \mathrm{e}^{-1}$ \\
\hline
\end{tabular}

الجدول(6) يوضح الفرق بين متوسط مربع الخطأ بطريقة الهوموتوبي التحليلية للمتسلسلة (50) وطريقة

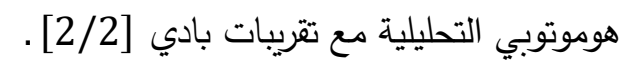

\begin{tabular}{|c|c|}
\hline MSE- HAM & $4.9989 \mathrm{e}^{-6}$ \\
\hline MSE - PA- HAM & $1.9627 \mathrm{e}^{-6}$ \\
\hline
\end{tabular}

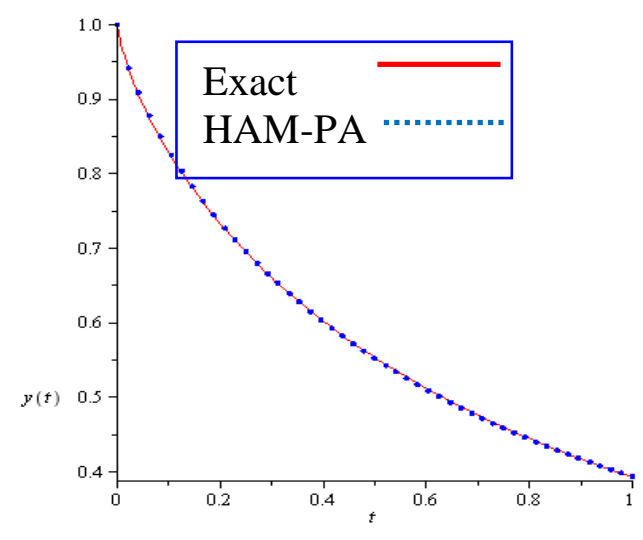

(b)

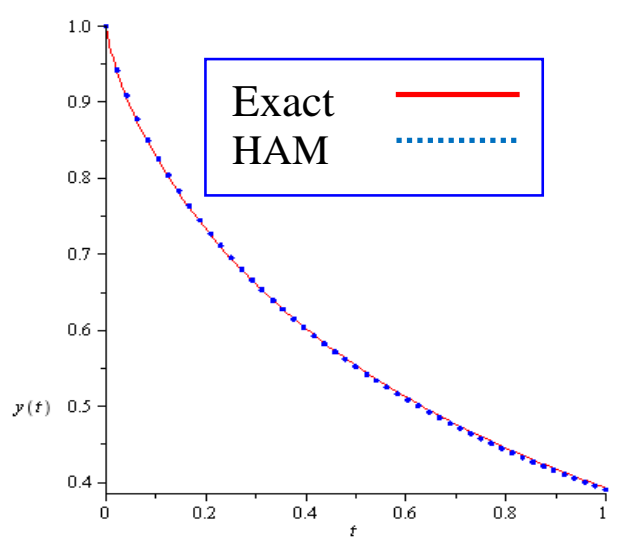

(a)

الثكل(3) : مقارنة بين الحل المضبوط والطرق المستخدمة عندما a

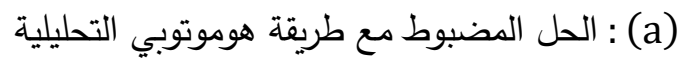

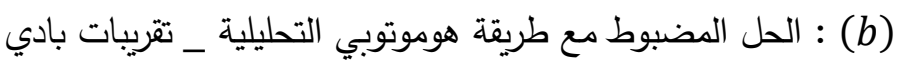


مثال (2) (20]):

لتكن لدينا مسألة القيم الابتدائية الخطية ذات الرتبة الكسرية $D^{0.5} y(t)=-y(t)+t^{2}+\frac{2}{\Gamma(2.5)} t^{1.5}$

مع الثرط الابتدائي

$y(0)=0$

والحل المضبوط للمسألة (53) هو

$y(t)=t^{2}$

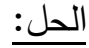

نفرض أن yo 0 = 0 وبالاستعانة بالمعادلة (35) نستطيع تركيب الهوموتوبي كالاتي : $R_{m-1}\left(\vec{y}_{m-1}(t)\right)=D^{0.5} y_{m-1}(t)+y_{m-1}(t)-\left(t^{2}+\frac{2}{\Gamma(2.5)} t^{1.5}\right)\left(1-x_{m}\right)$

الان باستعمال معادلة التشوه (deformation equation) من الرتبة m عندما 1 التصبح: $y_{m}(t)=\chi_{m} y_{m-1}(t)-\chi_{m} \sum_{j=0}^{0} y_{m-1}{ }^{(j)}\left(0^{+}\right) \frac{t^{j}}{j !}$
$+h J^{0.5}\left[R_{m-1}\left(\vec{y}_{m-1}(t)\right)\right]$

عندما 1 = 1 , بالتعويض في المعادلة (54) نحصل على :

$y_{1}(t)=\frac{-\Gamma(3) h t^{2.5}}{\Gamma(3.5)}-h t^{2}$

وعندما ...

$y_{m}(t)=y_{m-1}(t)+h\left[y_{m-1}(t)+J^{0.5} y_{m-1}(t)\right]$

بالتعويض عن $2=2$ في المعادلة والاستعانة بخصائص تكامل ريمان ليوفيل نحصل على :

$y_{2}(t)=y_{1}(t)+h\left[y_{1}(t)+J^{0.5} y_{1}(t)\right]$

$y_{2}(t)=-h(1+2 h) \frac{\Gamma(3) t^{2.5}}{\Gamma(3.5)}-h(1+h) t^{2}-\frac{h^{2} \Gamma(3) t^{4.5}}{\Gamma(5.5)}$ 
بالتعويض عن $3=3$ في المعادلة (55)نحصل على:

$$
\begin{aligned}
& y_{3}(t)=y_{2}(t)+h\left[y_{2}(t)+J^{0.5} y_{2}(t)\right] \\
& y_{3}(t)=-h\left(1+4 h+3 h^{2}\right) \frac{\Gamma(3) t^{2.5}}{\Gamma(3.5)}-h\left(1+2 h+h^{2}\right) t^{2}-\frac{h^{2}(2+3 h) \Gamma(3) t^{4.5}}{\Gamma(5.5)}- \\
& \frac{h^{3} \Gamma(3) t^{5.5}}{\Gamma(6.5)}
\end{aligned}
$$

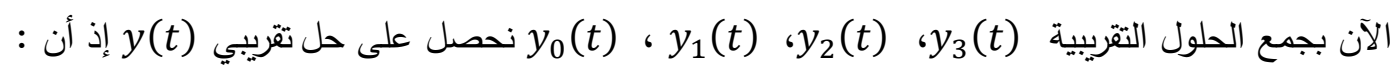

$$
\begin{aligned}
& y(t)=\sum_{m=0}^{3} y_{m}(t)=-h\left[1+(1+2 h)+\left(1+4 h+3 h^{2}\right) \frac{\Gamma(3) t^{2.5}}{\Gamma(3.5)}-\cdots-\right. \\
& \frac{h^{3} \Gamma(3) t^{5.5}}{\Gamma(6.5)}
\end{aligned}
$$

ثم بالتعويض عن

$y(t)$

$=5.8976 e^{-2} t^{3.5}-1.4699 e^{-1} t^{3}+1.1374 e^{-1} t^{2.5}$

$+9.7300 e^{-1} t^{2}$

بأخذ قيم مختلفة ذا وتعويضهم في متسلسلة الحل (57) نحصل على حلول تقريبية بطريقة هوموتوبي

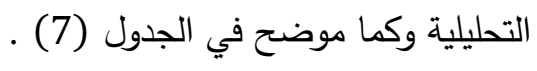

$$
\text { نفرض أن } x \text { = }
$$

$y^{*}(x)$

$=5.8976 e^{-2} x^{7}-1.4699 e^{-1} x^{6}+1.1374 e^{-1} x^{5}$

$+9.7300 e^{-1} x^{4}$

ألان نقوم بحل متسلسلة الحل الناتجة (x) على :

$[5 / 6]$

$=\frac{9.7300 e^{-1} t^{2}+5.4876 e^{-1} t^{2.5}}{1+4.4709 e^{-1} t^{0.5}+9.8815 e^{-2} t-4.6178 e^{-3} t^{1.5}-1.1630 e^{-2} t^{2}-5.3275 e^{-3} t^{2.5}-8.5452 e^{-4} t^{3}}$

بأخذ قيم مختلفة لـ t وتعويضهم في متسلسلة بادي (59) نحصل على حلول تقريبية بطريقة هوموتوبي التحليليلة

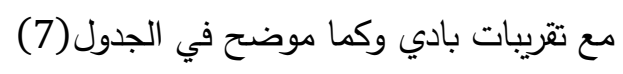


جدول (7): يتضمن نتائج الحل المضبوط , الحلول التقريبية بطريقة الهوموتوبي و الحلول التقريبية لمتسلسلة بادي (59)عند قيم t المختلفة.

\begin{tabular}{|c|c|c|c|}
\hline$t$ & Exact Solution & $\begin{array}{c}\text { Approximate } \\
\text { Solution using HAM }\end{array}$ & $\begin{array}{c}\text { Approximate } \\
\text { Solution using } \\
\text { HAM-PA }\end{array}$ \\
\hline 0 & 0 & 0 & 0 \\
\hline 0.1 & $1 e^{-2}$ & $9.9613 e^{-3}$ & $9.9613 e^{-2}$ \\
\hline 0.2 & $4 e^{-2}$ & $3.9989 e^{-2}$ & $3.9989 e^{-2}$ \\
\hline 0.3 & $9 e^{-2}$ & $9.0080 e^{-2}$ & $9.0080 e^{-2}$ \\
\hline 0.4 & $1.6 e^{-1}$ & $1.6016 e^{-1}$ & $1.6016 e^{-1}$ \\
\hline 0.5 & $2.5 e^{-1}$ & $2.5019 e^{-1}$ & $2.5019 e^{-1}$ \\
\hline 0.6 & $3.6 e^{-1}$ & $3.6011 e^{-1}$ & $3.6012 e^{-1}$ \\
\hline 0.7 & $4.9 e^{-1}$ & $4.8990 e^{-1}$ & $4.8992 e^{-1}$ \\
\hline 0.8 & $4.6 e^{-1}$ & $6.3957 e^{-1}$ & $6.3961 e^{-1}$ \\
\hline 0.9 & $8.1 e^{-1}$ & $8.0915 e^{-1}$ & $8.0924 e^{-1}$ \\
\hline 1.0 & 1 & $9.9871 e^{-1}$ & $9.9887 e^{-1}$ \\
\hline
\end{tabular}

الجدول(8) يوضح الفرق بين متوسط مربع الخطأ بطريقة الهوموتوبي التحليلية للمتسلسلة (57) وطريقة

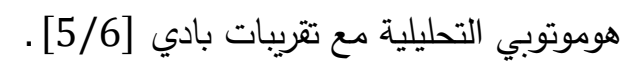

\begin{tabular}{|c|c|}
\hline MSE- HAM & $2.3956 e^{-7}$ \\
\hline MSE - PA- HAM & $1.8986 e^{-7}$ \\
\hline
\end{tabular}

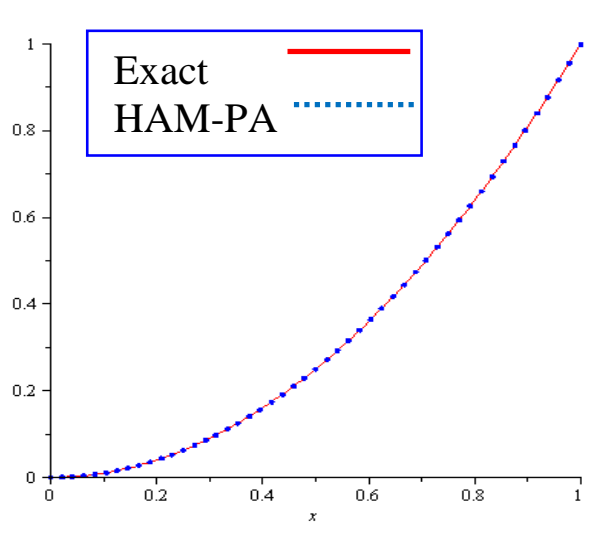

(b)

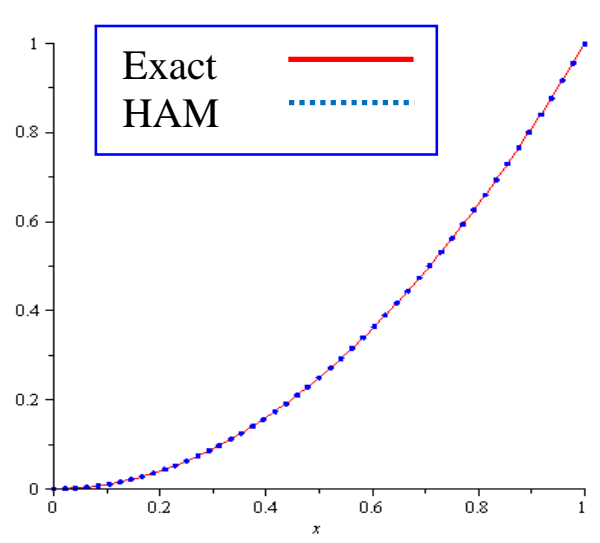

(a)

الثكل(4) : مقارنة بين الحل المضبوط والطرق المستخدمة عندما

(a) : الحل المضبوط مع طريقة هوموتوبي التحليلية

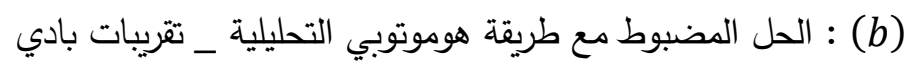

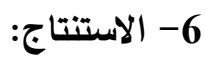

1- في هذا البحث أثبتتا كفاهه طريقة هوموتوبي التحليلية مع تقريبات بادي لحل مسأئل القيم الابتدائية ذات الرتب الكسرية إذ اظهرت النتائج مدى إمكانية تقريبات بادي لتحسين نتائج طريقة هوموتوبي التحليلية .

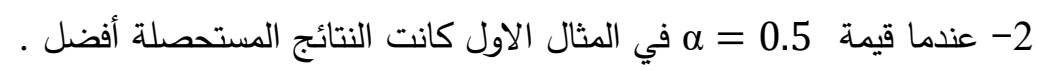
3- تقريب بادي يحل فقط المتسلسلات ذات ألاسس الطبيعية ولذلك استخدمنا الفرضية عدد كسري المذكوره في البند الرابع التحويل المتسلسلة ذات الاسس الكسرية الى اسس طبيعية. 
[1] Bhrawy, A. H., and Alghamdi, M., A. B. V P's, 2012(1), 62(2012).

[2] Choudhury, M. D., Chandra, S., Nag, S., Das, S., and Tarafdar, S.. Physicochemical and Engineering Aspects, 407, 64-70(2012).

[3] Flandoli, F., and Ciprian A.T. Journal of Functional Analysis 258.1 :279-306 (2010)

[4] Ghomashi, A., Soheil S. and Hakimzadeh A. Journal of Intelligent \& Fuzzy Systems 26:367-378(2014).

[5] Magin, R. L., Ingo, C., Colon-Perez, L., Triplett, W., and Mareci, T. H. Microporous and Mesoporous Materials, 178, 39-43 (2013).

[6] Bhrawy, A., Alhamed, Y., Baleanu, D. and Al-Zahrani, A. Fractional Calculus and Applied Analysis, 17(4), 1137-1157(2014).

[7] Liao, Sh J. Ph. D. Thesis, Shanghai Jiao Tong University (1992).

[8] Noor, M. A. and Syed Tauseef Mohyud-Din. International Journal of Nonlinear Science8.1: 27- 31(2009).

[9] Jafari H. and Seifi, S. Communications in Nonlinear Science and Numerical Simulation, 14(5), 2006-2012 (2009).

[10] Mohammed, O. H. Journal of Al-Nahrain University, 13, 149-155(2010).

[11] Momani, S. and Zaid O. Journal of Computational and Appl. Math. 207.1: 96110(2007)

[12] Zurigat M., Momani S., and Alawneh A. Computers and Mathematics with Applications, 59(3), 1227-1235(2010).

[13] Bhrawy, A., Alhamed, Y., Baleanu, D., and Al-Zahrani, A. Fractional Calculus and Applied Analysis, 17(4), 1137-1157(2014).

[14] Singh, Brajesh K, and Pramod K. arXiv preprint arXiv:1611.07171 (2016).

[15] Ogunlaran, O. M. and Sagay-Yusuf H. British Journal of Mathematics \& Computer Science 14.3: 1(2016).

[16] Drucker, H., Burges, C. J., Kaufman, L., Smola, A. J., and Vapnik, V.. ,In Advances in neural information processing systems (pp. 155-161) (1997) .

[17] Al-Hayani, W., Alzubaidy, L., and Entesar, A. Appl. Math, 11(2), 407416(2017).

[18] Odibat, Z. and El-ajou A. IAENG International Journal of Applied Math 40.2 (2010).

[19] Esmaeili, S., Shamsi, M., and Luchko, Y. Computers and Mathematics with Applications, 62(3), 918-929(2011).

[20] Pandey R.K., Bhardwaj A. B. H. I. N. A. V and Syam M., J. Fract. Calc. Appl 5.1: 129-145(2014). 